\title{
VITAMIN D TOXICITY \& UNDETECTABLE SERUM LEVELS - A CONUNDRUM
}

Vimal Venugopal, Miles J Levy, Narendra L Reddy, Faizanur Rahman and Ragini C Bhake

University Hospitals Leicester NHS Trust Kingdom

\section{Presenting history}

A 59 year old lady presented to the acute medical unit in May 2017 after being referred by her general practitioner for symptomatic hypercalcaemia. This had been picked up as part of monitoring bloods for an alternative medicine regimen.

She had a history of Multiple Sclerosis diagnosed in 1998, she had a relapsing remitting presentation initially and she was started on disease modifying therapy started on 2004, which she discontinued after a year partly due to needle phobia. She stopped having relapses but had problems with a gradual decline in mobility as well as spasms and urinary urgency. She was treated symptomatically by the local neurology service.

She had found an alternative medicine regimen - "The Coimbra Protocol"1 -which is used to attempt to stop the progression of multiple sclerosis. This is a regimen which uses high doses of

colecalciferol in combination with other supplements which include magnesium, Vitamin B2, Vitamin B5, omega 3 fatty acids. She had been consulting a nutritionist privately in Ireland who had been requesting regular blood tests as part of monitoring serum calcium levels and advising on dose adjustments. She had been advised to drink at least 3 litres of water a day.

She had started taking 10000 international units of colecalciferol daily since December 2016, her dosage had increased to $170000 \mathrm{IU}$ once daily since March 2017.

In the 1 week leading up to her admission, she stated she had reduced her fluid intake to 2 litres/day and had been feeling generally unwell.

Her blood test by her GP immediately prior to admission in May 2017 showed her serum calcium to be $3.66 \mathrm{mmol} / \mathrm{l}$ (normal range $2.2-2.6 \mathrm{mmol} / \mathrm{l}$ ) and subsequently she was referred to hospital. All her previous calcium levels had been in the normal range. The initial blood test in hospital showed a serum calcium of $3.76 \mathrm{mmol} / \mathrm{l}$.

\begin{tabular}{|l|l|}
\hline Medication & Dose \\
\hline Colecalciferol & $\begin{array}{l}\text { Date } \\
\text { daily }\end{array}$ \\
\hline Pre-activated & $6.4 \mathrm{mg}$ once daily
\end{tabular}

Vitamin B2

Omega 3 fatty 3 tabs once daily acid supplements

Magnesium 2 tabs once daily

Citrate

Solifenacin

10mg once daily

Lactulose

$10 \mathrm{ml}$ twice daily

Sennoside

$7.5 \mathrm{mg}$ once daily

Medication history at time of presentation

\section{Inpatient history}

Parathyroid hormone level was checked at time of admission, result was $4.7 \mathrm{pmol} / \mathrm{l}$ (normal range 1.6-7.5). Serum 25hydroxy Vitamin D as measured by immunoassay (at our local laboratory) was reported as $<15 \mathrm{nmol} / \mathrm{L}$ which was not in keeping with history of ingestion of very high doses of colecalciferol.

In light of above results, myeloma screen and serum ACE (screening for sarcoidosis) were sent and returned negative. Computed tomography scans of her thorax, abdomen and pelvis was performed to investigate for a malignant aetiology, the only finding was that of an adrenal incidentaloma.

\section{Treatment for the}

hypercalcaemia was with vigorous rehydration with intravenous $0.9 \% \mathrm{NaCl}$ and stopping her vitamin D supplementation.

Her serum calcium had reduced to $2.64 \mathrm{mmol} / /$ six days post admission. Patient was

discharged once all investigations above had been performed and patient was symptomatically better. She had expressed a wish to restart Vitamin D supplementation but she was asked not to restart Vitamin D supplementation until post discharge bloods showed that her calcium had normalised and her Vitamin D levels were coming down.

As the serum 25 hydroxy Vitamin D levels were not in keeping with history, bloods were sent off to a different laboratory.

\begin{tabular}{l|l|l|l|l}
$\begin{array}{l}\text { Adjusted } \\
\text { calcium }\end{array}$ & $\begin{array}{l}\text { Phosphate } \\
(\mathrm{mmol} / \mathrm{l})\end{array}$ & $\begin{array}{l}\text { Creatinine } \\
\text { (micromol }\end{array}$ & $\begin{array}{l}\text { Vitamin D } \\
(\mathrm{nmol} / \mathrm{l})\end{array}$ & $\begin{array}{l}\text { PTH } \\
\text { (pmol/l) }\end{array}$ \\
$\begin{array}{l}\text { (mmol/l) } \\
\text { NR (0.8- }\end{array}$ & /I) NR (60- & & NR (1.6- \\
NR (2.2- & $1.5)$ & $120)$ & & $7.5)$ \\
$2.6)$ & & & & \\
\hline
\end{tabular}

$27 / 04 / 2016 \quad 2.34$

1.28

52

112

$03 / 02 / 20172.33$

1.28

56

100

$08 / 05 / 20173.66$

0.90

111

09/05/2017 3.76

0.93

90

$11 / 5 / 2017 \quad 3.23$

0.70

71

$13 / 5 / 2017 \quad 2.96$

0.72

0.82

61

$15 / 5 / 2017 \quad 2.64$

0.82

42

$02 / 06 / 2017$

$30 / 6 / 2017$

2.52

$>374$

47

Post discharge of the patient, we received results from the external laboratory which gave a result of $862 \mathrm{nmol} / \mathrm{I}$ (99.6\% Vitamin D3). Thus the results from our local laboratory were looked at again, it emerged that the actual result was $>374 \mathrm{nmol} / \mathrm{l}$, but an information technology (IT) error had actually caused the very high result to default to a $<15 \mathrm{nmol} / \mathrm{l}$. As such extremely high vitamin $D$ levels had not seen before this IT error had not been picked up previously.

\section{Discussion}

Vitamin D toxicity can occur secondary to excessive supplementation of Colecalciferol or more commonly, its metabolites Calcitriol and Alfacalcidol. The toxicity presents as hypercalcaemia with characteristic symptoms such as nausea, vomiting, irritability and weakness.

Despite widespread use of colecalciferol supplementation, toxicity rarely occurs ${ }^{2,3}$. Local guidelines to treat severe Vitamin D deficiency suggest a regimen of 10000 IU daily for two months and then 1000-2000 IU daily as maintenance. Public Health England guidelines ${ }^{4}$ suggest supplementation of 10 micrograms (400 IU) daily for all adults through Autumn and Winter, and supplementation of $400 \mathrm{IU}$ all year round for at risk adults.

Thus in this case, it is important to note that the dosage of up to 170000 IU daily is far in excess of usual recommended dosages for treatment and prophylaxis of Vitamin D deficiency.

Interestingly the parathyroid hormone level was in the normal range despite the elevated serum calcium levels.
Reviewing the case reports of Vitamin D toxicity, a similar picture of non suppressed PTH (in the normal range) has been reported on multiple occasions ${ }^{5,6,7}$. The normal PTH level in combination with the initial reported undetectable level of 25 hydroxyVitamin D led us to investigate

Learning points

Although recommended dose of Vitamin $D$ have not shown to cause toxicity, patients may be taking significantly higher doses thanr recommended in alternative medicine regimens and thus toxicity can occur with the use of colecaclciferol over the counter supplements.

Parathyroid hormone can often be normal in the setting of Vitamin D3 toxicity, this maybe due to a slower rise in serum calcium than in other causes of hypercalcaemia.

When blood test results are not in keeping with the clinical picture, liaison with clinical chemistry teams can help clarify the situation especially in this case as a simple reporting error was the issue.

References:

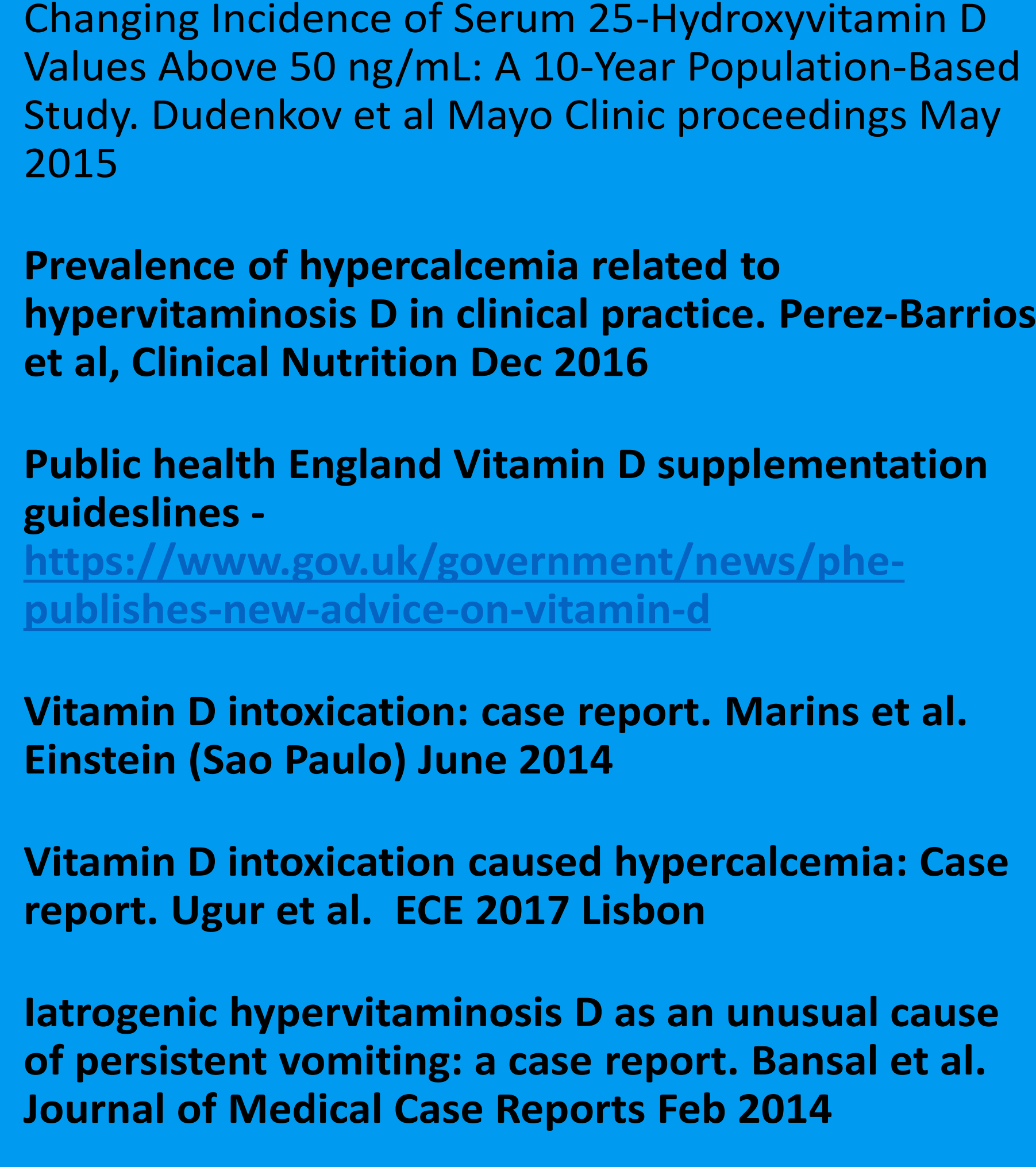
other causes of hypercalcaemia. 